\title{
Goniometer-apps in hand surgery and their applicability in daily clinical practice
}

\author{
Paul Kuegler ${ }^{1 *}$, Paul Wurzer ${ }^{1}$, Alexandru Tuca', Gerald Sendlhofer ${ }^{1,2}$, David Benjamin Lumenta',
} Michael Giretzlehner ${ }^{3}$ and Lars-Peter Kamolz ${ }^{1}$

\begin{abstract}
Background: Smartphones have become an integral part of our daily lives, and numerous applications (apps) make use of the goniometer, which is comparable to a medical protractor. The aim of this study was to evaluate the availability, reliability and validity of medical apps using the goniometer function in smartphones.

Methods: Sixteen goniometer-apps downloaded from Apple's (twelve apps) and Google's (four apps) app stores matched initial inclusion criteria (inclusion criteria: * $g o n i{ }^{*}$ or * ${ }^{*}$ om*, specifically designed to measure angles) were assessed and compared to a universal mechanical goniometer by measuring an irregular, four-sided pyramid with distinct angles.

Results: Twelve iOS (Apple's operating system) and four Android (Google's operating system) apps were included. Ten of twelve iOS apps were showing an average deviation from the real angles of a maximum $1.8^{\circ}$; the values for Android apps did not exceed $1.3^{\circ}$.

Conclusions: Fourteen out of sixteen apps were reliable and valid for measuring angles. Based on correct handling and adequate user-applicability of the on-board goniometer these apps can make a smartphone applicable for data acquisition in medicine. So far, smartphone apps for measuring angles have not been readily applied in clinical practice in hand surgery.
\end{abstract}

Keywords: Goniometer, Smartphones, Apps, iOS, Android

\section{Background}

Worldwide sales of smartphones were estimated at 986 million units in $2013(+42.3 \%$ as compared to 2012), and accounted for $53.6 \%$ of total mobile phone sales in 2013. With a share of $31.0 \%$ Samsung dominates the smartphone market followed by Apple (15.6\%) and other manufacturers accounting for $53.4 \%$ with each of them not exceeding 5\%. Google's Android has a 78.4\% market share of the operating systems, followed by Apple's iOS with $15.6 \%$ and the remainder (eg. Microsoft, Blackberry) with approximately $6 \%[1]$.

In 2013 both Apple's App Store and Google's Play Store offered more than one million apps surpassing 50 billion downloads [2,3]. Recent estimates anticipate over 500 million users applying smartphones in health-care related contexts by 2015 [4] with a steadily increasing availability [5-8].

A goniometer is commonly used in hand surgery to assess the range-of-motion of joints. Different types of goniometers exist, and start from simple universal mechanic devices, advance to goniometers with two adjustable arms, gyroscope-based tools [9] and even go to sophisticated electromechanical systems with sensors integrated in fabrics [10].

The aim of this study was to investigate apps using the goniometer-function in smartphones, to analyze their availability, reliability and validity versus a universal manual medical-grade goniometer, and to evaluate the price tag and approval for use in Medicine based on the Medical Device Act of Austria.

* Correspondence: kuegler.paul@gmail.com

'Division of Plastic, Aesthetic and Reconstructive Surgery, Department of

Surgery, Medical University of Graz, Graz, Austria

Full list of author information is available at the end of the article 


\section{Methods}

The apps from each store were downloaded by use of an iPhone $5^{\circ}$ and a Samsung Galaxy $2^{\circ}$ running iOS 7.1.1 and Android 4.1.2, respectively.

The search for apps in each store (iOS in Apple's App Store on 30 May 2014, Android in Google's Play Store on 02 June 2014) was performed using the expression "gonio*. Additionally, all apps labeled with the term *range of motion* or *ROM* were also included.

All apps not designed for the use in medicine were excluded (e.g. bubble level apps, goniometer in the field of stereophony).

A manual goniometer served as control (universal mechanical goniometer by Prestige Medical $^{\oplus}$, Northridge, California, USA).

For measurements, we used a reference object with an irregular, four-sided pyramidal shape that was specially designed and built for this study. Constructing the pyramid was done as follows: the measures were first drawn with the AutoCAD ${ }^{\circ}$ (version 2015, Autodesk inc., San Rafael, California, USA) software and subsequently built on a 1:1 scale. Table 1 contains all side lengths of the pyramid. Four angles formed by the pyramid's faces were selected for the measurements, and are indicated in Table 2. An angle was defined by the two faces forming it, and a face was defined by three points. In order to improve the illustration each edge between two faces was colored. The colored edges are depicted in Fig. 1. These edges were used to measure the angles.

Measurements were repeated five times per angle per app, resulting in 20 measurements for each app by one trained user.

Apart from the price tag invoked per app, we checked whether each software/hardware had been approved by an appointed authority as explained in the Medical Device Act (Austria), where the following terms for definition as Medical Device apply ( $\$ 2$ definition of terms): "Medical devices are all [...], software, [...], intended by the manufacturer to be used for human beings, by virtue of their functions, for the purpose of a) diagnosis,

Table 1 Lengths of the pyramid

\begin{tabular}{lc}
\hline Side & Length (in cm) \\
\hline$A B$ & 12,7 \\
$B C$ & 9,5 \\
$C D$ & 10,8 \\
$A D$ & 18,6 \\
$A E$ & 14,0 \\
$B E$ & 13,2 \\
$C E$ & 12,1 \\
DE & 15,0 \\
$E^{\prime} E$ (=height) & 10,0 \\
\hline
\end{tabular}

Table 2 Measured angles

\begin{tabular}{lllr}
\hline Angle & Color of the Edge & Greek Letter & Value \\
\hline$\varangle A D E, A B E$ & Red & $a$ & $90,8^{\circ}$ \\
$\varangle B A E, B C E$ & Blue & $\beta$ & $105,2^{\circ}$ \\
$\varangle C B E, C D E$ & Green & $\gamma$ & $145,1^{\circ}$ \\
$\varangle D C E, D A E$ & Yellow & $\delta$ & $79,4^{\circ}$ \\
\hline
\end{tabular}

prevention, monitoring, treatment or alleviation of disease, b) diagnosis, monitoring, treatment, alleviation or compensation of injuries or handicaps, [...]."

\section{Statistical analysis}

Data were expressed in means $+/$ - standard deviation. Deviations from the control value were expressed in degrees of arc, and apps with less than 3 degrees deviation were considered acceptable.

\section{Results}

Fourteen apps in Apple's App Store contained the term "gonio", two apps the term "ROM* and one app called "PT Tools" made use among other functions of the goniometer.

Five apps were excluded: one was a duplicate (appearing as iPad and iPhone version, the iPad version was excluded), another was a goniometer for use in stereophony ("MC Goniometer"), and three apps ("Forearm Goniometer", "Frozen Shoulder Goniometer" and "Knee Goniometer") were only applicable in the named joint regions and therefore not suitable for measurements of the pyramid.

The remaining twelve apps were split into two functional groups: apps using the internal accelerometer (a sensor used to determine a smartphone's spatial position) and apps measuring joint angles on images. In the latter, the desired angle was marked on a camera picture or uploaded photograph, and the angles were then calculated by the app (Table 3 ).

Twenty apps in Google's Play Store contained the term "gonio*, and one app the term *rom".

Seventeen apps were excluded: five apps containing "gonio" ("Goniometric Compass", "Herrscher Goniometer", the Chinese Version of "Goniometer", "Goniometer Pro" available as trial and full version and "Free Goniometer") were only suitable to measure head movements. The other twelve apps did not contain "gonio* or *rom* (although they had appeared in the search), but were not suited for measurements.

The remaining four apps are listed in Table 4.

Fourteen of a total of sixteen investigated apps demonstrated less than $3^{\circ}$ deviation compared to the mechanical goniometer as well as the actual angles of the pyramid.

Ten of twelve iOS apps showed an average deviation from the actual angles of a maximum $1.8^{\circ}$ and the average deviation for Android apps did not exceed $1.3^{\circ}$. 


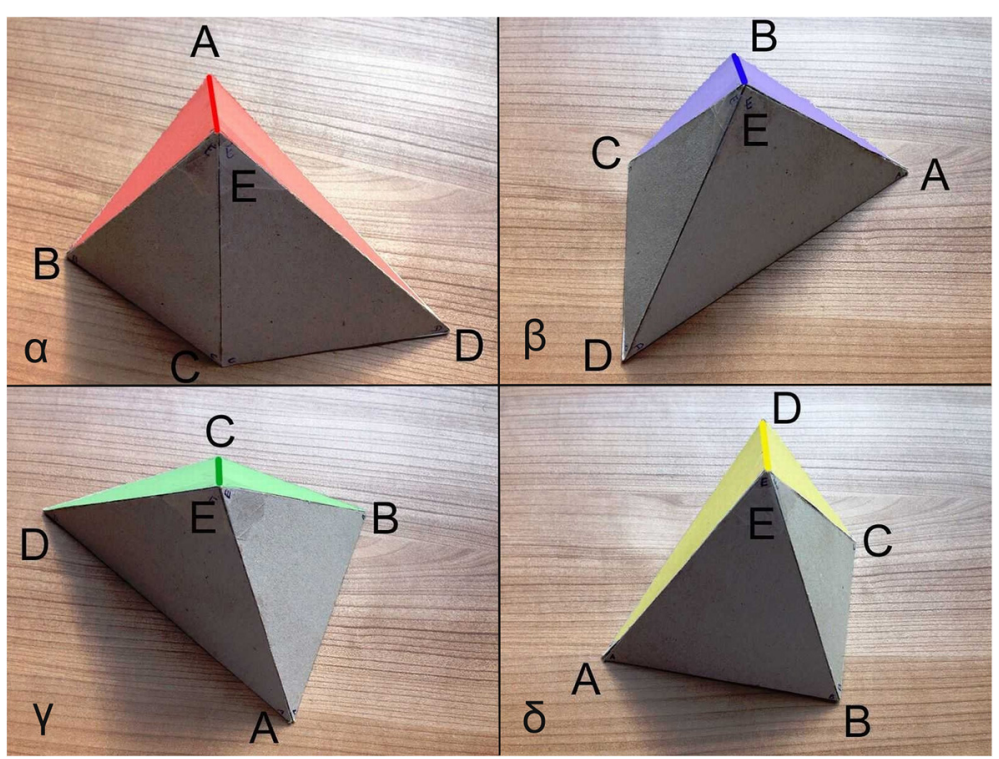

Fig. 1 The four measured angles $a, \beta, \gamma$ and $\delta$

When taking a closer look at the mean values for each angle separately, there was no iOS app exceeding $2.3^{\circ}$ and no Android app showed a higher deviation than $2.9^{\circ}$. Except for the two outliers, all results of iOS (Apple) and Android (Samsung) devices showed less than $3^{\circ}$ deviation.

Apps representing outliers were iOS apps "Goniometer" and "iGonio" with a measuring error of about $10^{\circ}$ at angle $\gamma$.

Detailed measuring results for all apps are listed in Table $\mathbf{5}$ and illustrated in a diagram in Fig. 2.

Ten of twelve products from Apple's App Store required a payment and the average price of an app (including the two free apps) was $4 €$. Of the four apps from Google's Play Store one was for free, one cost $0.96 €$, one

Table 3 Used iOS apps including price and functional principle

\begin{tabular}{llrl}
\hline Name & Developer & Price & $\begin{array}{l}\text { Functional } \\
\text { Principle }\end{array}$ \\
\hline Angulus ROM & MerginglT & $4.49 €$ & Accelerometer \\
GetMyROM & $\begin{array}{l}\text { Interactive Medical } \\
\text { Productions } \\
\text { Goniometer }\end{array}$ & $2.69 €$ & Accelerometer \\
Goniometer Pro & 5 uf5 & $4.49 €$ & Accelerometer \\
Goniometer & Ashok Shyam & $13.99 €$ & Accelerometer \\
Records & Free & Accelerometer \\
Goniometro & Antinio Costantino & $1.79 €$ & Accelerometer \\
Joint Goniometry & Diomedes Papas & $4.49 €$ & Accelerometer \\
Simple Goniometer & Ockendon.net & $0.89 €$ & Accelerometer \\
DrGoniometer & CDM & $10.99 €$ & Photo \\
iGonio & Abdel Zemirline & Free & Photo \\
PhotoGoniometer & Robert Branstrom & Free & Photo \\
PT Tools & David Raney & $3.59 €$ & Photo \\
\hline
\end{tabular}

required a payment of $0.77 €$ after 25 free measurements and "Goniometer Pro" cost 10.92€ (price of the same app in Apple's App Store was 13.99€).

None of the apps contained any reference stating that an authority investigated or approved any of the products, neither in the developer's country nor anywhere else.

\section{Discussion}

Fourteen of a total of sixteen apps were comparable to the mechanical goniometer and therefore reliable and valid for measuring angles. Two types of apps can be distinguished: apps using the smartphone's built-in accelerometer and apps working with images, so called photogoniometers. No substantial differences in reliability and validity were observed between these two groups. The benefit of photogoniometers is the possibility of taking a picture for measurements and simultaneously storing it for documentation (e.g. only bearing the patient's joint without further identification made possible).

To obtain correct angles, adequate positioning of the smartphone must be maintained according to each developer's instructions. Not every app measured angles in

Table 4 Used Android apps including price and functional principle

\begin{tabular}{llll}
\hline Name & Developer & Price & Functional Principle \\
\hline Goniometer Pro & 5 uf5 & $10.92 €$ & Accelerometer \\
Goniometer Records & $\begin{array}{l}\text { Indian Orthpaedic } \\
\text { Research Group }\end{array}$ & $0.77 €$ & Accelerometer \\
ROM Messung & YMED & Free & Accelerometer \\
Goniometer & T-Software & $0.96 €$ & Photo \\
\hline
\end{tabular}


Table 5 Measuring results (unit = degrees of arc, StaDev = standard deviation)

\begin{tabular}{|c|c|c|c|c|c|c|c|c|}
\hline Tool/App & OS & Function & Value & $a=90.8$ & $\beta=105.2$ & $Y=145.1$ & $\delta=79.4$ & Mean \\
\hline Mechanical & & & Mean & 90.8 & 105 & 145 & 79.8 & \\
\hline \multirow[t]{2}{*}{ Goniometer } & & & StaDev & 0.45 & 0 & 0 & 0.45 & 0.23 \\
\hline & & & Mean - True Value & 0 & -0.2 & -0.1 & 0.4 & 0.18 \\
\hline \multirow[t]{3}{*}{ Angulus ROM } & iOS & Sensor & Mean & 90.2 & 105.2 & 145.4 & 80.4 & \\
\hline & & & StaDev & 0.84 & 0.45 & 0.55 & 0.55 & 0.6 \\
\hline & & & Mean - True Value & -0.6 & 0 & 0.3 & 1 & 0.48 \\
\hline \multirow[t]{3}{*}{ GetMyROM } & iOS & Sensor & Mean & 90.38 & 106.5 & 146.72 & 81.7 & \\
\hline & & & StaDev & 0.31 & 0.27 & 0.34 & 0.33 & 0.31 \\
\hline & & & Mean - True Value & -0.42 & 1.3 & 1.62 & 2.3 & 1.41 \\
\hline \multirow[t]{3}{*}{ Goniometer } & iOS & Sensor & Mean & 89.6 & 107.4 & 155.4 & 79 & \\
\hline & & & StaDev & 0.55 & 0.55 & 0.55 & 1 & 0.66 \\
\hline & & & Mean - True Value & -1.2 & 2.2 & 10.3 & -0.4 & 3.53 \\
\hline \multirow[t]{3}{*}{ Goniometer Pro } & iOS & Sensor & Mean & 90 & 106.4 & 145.6 & 81.2 & \\
\hline & & & StaDev & 0 & 0.55 & 0.55 & 0.84 & 0.49 \\
\hline & & & Mean - True Value & -0.8 & 1.2 & 0.5 & 1.8 & 1.08 \\
\hline \multirow[t]{3}{*}{ Goniometer Records } & iOS & Sensor & Mean & 90 & 105.6 & 146.4 & 79.8 & \\
\hline & & & StaDev & 0 & 0.55 & 0.55 & 0.45 & 0.39 \\
\hline & & & Mean - True Value & -0.8 & 0.4 & 1.3 & 0.4 & 0.73 \\
\hline \multirow[t]{3}{*}{ Goniometro } & iOS & Sensor & Mean & 88.62 & 106.8 & 146.42 & 80.24 & \\
\hline & & & StaDev & 0.45 & 0.26 & 0.39 & 0.4 & 0.38 \\
\hline & & & Mean - True Value & -2.18 & 1.6 & 1.32 & 0.84 & 1.49 \\
\hline \multirow[t]{3}{*}{ Joint Goniometry } & iOS & Sensor & Mean & 90.32 & 106.26 & 145.58 & 81.46 & \\
\hline & & & StaDev & 0.15 & 0.27 & 0.28 & 0.38 & 0.27 \\
\hline & & & Mean - True Value & -0.48 & 1.06 & 0.48 & 2.06 & 1.02 \\
\hline \multirow[t]{3}{*}{ Simple Goniometer } & iOS & Sensor & Mean & 90.4 & 105.4 & 145.4 & 79.6 & \\
\hline & & & StaDev & 0.55 & 0.55 & 0.55 & 0.55 & 0.55 \\
\hline & & & Mean - True Value & -0.4 & 0.2 & 0.3 & 0.2 & 0.28 \\
\hline \multirow[t]{3}{*}{ DrGoniometer } & $\mathrm{iOS}$ & Photo & Mean & 89.96 & 103.14 & 144.68 & 81.26 & \\
\hline & & & StaDev & 0.75 & 1.02 & 1.2 & 0.75 & 0.93 \\
\hline & & & Mean - True Value & -0.84 & -2.06 & -0.42 & 1.86 & 1.3 \\
\hline \multirow[t]{3}{*}{ ¡Gonio } & iOS & Photo & Mean & 88.4 & 109 & 155.4 & 74.8 & \\
\hline & & & StaDev & 1.95 & 2.74 & 1.14 & 1.48 & 1.83 \\
\hline & & & Mean - True Value & -2.4 & 3.8 & 10.3 & -4.6 & 5.28 \\
\hline
\end{tabular}


Table 5 Measuring results (unit = degrees of arc, StaDev = standard deviation) (Continued)

\begin{tabular}{|c|c|c|c|c|c|c|c|c|}
\hline \multirow[t]{3}{*}{ PhotoGoniometer } & $\mathrm{iOS}$ & Photo & Mean & 89.8 & 103.2 & 143.2 & 81.6 & \\
\hline & & & StaDev & 1.3 & 1.3 & 0.84 & 0.55 & 1 \\
\hline & & & Mean - True Value & -1 & -2 & -1.9 & 2.2 & 1.78 \\
\hline \multirow[t]{3}{*}{ PT Tools } & iOS & Photo & Mean & 89.4 & 102.2 & 145.4 & 80.8 & \\
\hline & & & StaDev & 1.14 & 0.84 & 0.55 & 0.45 & 0.75 \\
\hline & & & Mean - True Value & -1.4 & -3 & 0.3 & 1.4 & 1.53 \\
\hline \multirow[t]{3}{*}{ Goniometer Pro } & Android & Sensor & Mean & 89.6 & 105.2 & 142.8 & 81 & \\
\hline & & & StaDev & 0.55 & 1.3 & 0.84 & 0.71 & 0.85 \\
\hline & & & Mean - True Value & -1.2 & 0 & -2.3 & 1.6 & 1.28 \\
\hline \multirow[t]{3}{*}{ Goniometer Records } & Android & Sensor & Mean & 90.8 & 105.2 & 142.2 & 80 & \\
\hline & & & StaDev & 0.84 & 0.84 & 1.3 & 0.71 & 0.92 \\
\hline & & & Mean - True Value & 0 & 0 & -2.9 & 0.6 & 0.88 \\
\hline \multirow[t]{3}{*}{ ROM Messung } & Android & Sensor & Mean & 90.6 & 105.6 & 145 & 80.8 & \\
\hline & & & StaDev & 0.55 & 0.89 & 0.71 & 0.84 & 0.75 \\
\hline & & & Mean - True Value & -0.2 & 0.4 & -0.1 & 1.4 & 0.53 \\
\hline \multirow[t]{3}{*}{ Goniometer } & Android & Photo & Mean & 90.4 & 104.4 & 145.6 & 80.8 & \\
\hline & & & StaDev & 1.14 & 0.89 & 1.34 & 1.92 & 1.32 \\
\hline & & & Mean - True Value & -0.4 & -0.8 & 0.5 & 1.4 & 0.78 \\
\hline
\end{tabular}




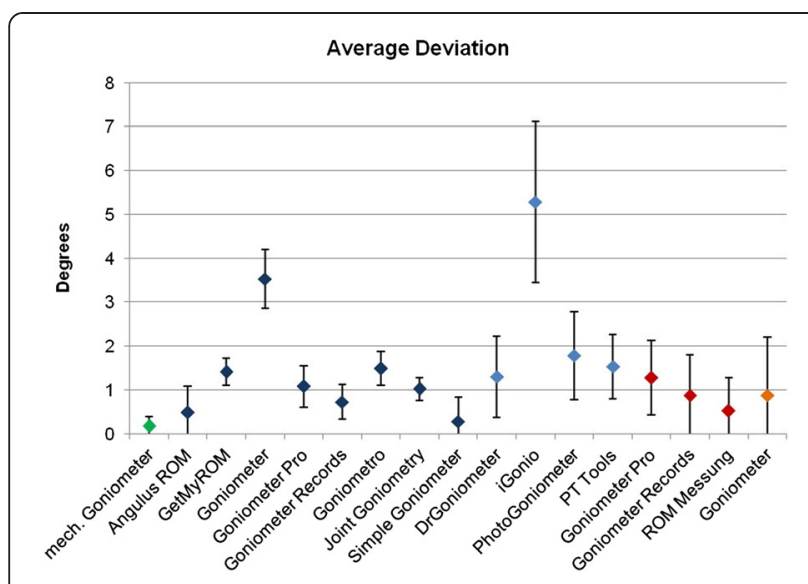

Fig. 2 Average deviation of mean value from true value

the same way (e.g. while some of them need to be put up along the long edge, others were required to be put flat on the back side). Fig. 3 illustrates the use of an accelerometerapp in comparison to the manual goniometer.

None of the apps was approved by an appointed authority. Apps may still be used in clinical practice under certain circumstances and precautions (e.g. for simple measuring purposes). As part of the conformity assessment procedure a medical device is allocated to a risk class depending on its potential risk exerted on human beings. Manufacturers can attribute products to class I in their sole responsibility (measuring function (Im) or sterile aspect (Is) checked by an appointed authority). For class III products successful passing of clinical trials is mandatory for market approval.

However, the application of software apps without approval as medical product carries some risks: if no instructions, no risk analyses, no warnings, no contact persons in case of problems or queries, no responsible contact person(s) of a company are listed, there is therefore no support in case of a (critical) incident available. If the developer or manufacturer is not registered as medical product manufacturer with an appropriate authority, the user should be aware that he/she may be using the product (app) in sole personal responsibility.

With regard to medical grade hygienic standards, wipe disinfection can be carried out for mechanical goniometers as well as smartphones, which can also be placed in a sterile plastic bag.

Only a few studies evaluated goniometer apps in clinical studies. Ferriero et. al were investigating the iOS photogoniometer "DrGoniometer" in 2011 and 2013. In the former, the app was compared to a standard mechanical goniometer and judged reliable [11]. In the latter, the authors assessed in the same methodical setting angles in a knee joint, drawing the same conclusions [12]. Both studies assessed intra-rater and inter-rater variability. 2014 Mitchell et. al were comparing iOS apps "GetMyROM" and "DrGoniometer" and found both reliable and comparable to the mechanical goniometer [13]. In another study testing the iOS accelerometer "Simple Goniometer", Jones et al. judged the app reliable [14]. The four clinical studies support the results of this study with regard to the apps "DrGoniometer", GetMyROM" and "Simple Goniometer". The 'knee goniometer' [15] as well as other programs-not tested in our study-were also confirmed to obtain reliable results in earlier reports [16-18]. Interestingly, we did not retrieve clinical studies in hand surgery dealing with the other thirteen apps tested in our study, and so far no Android app was evaluated in a clinical setting.

One limitation was that the study was conducted in a preclinical setting on a standardized pyramid to assess validity. In a clinical setting, no reproducible straight lines and edges can be found as compared to our setup.

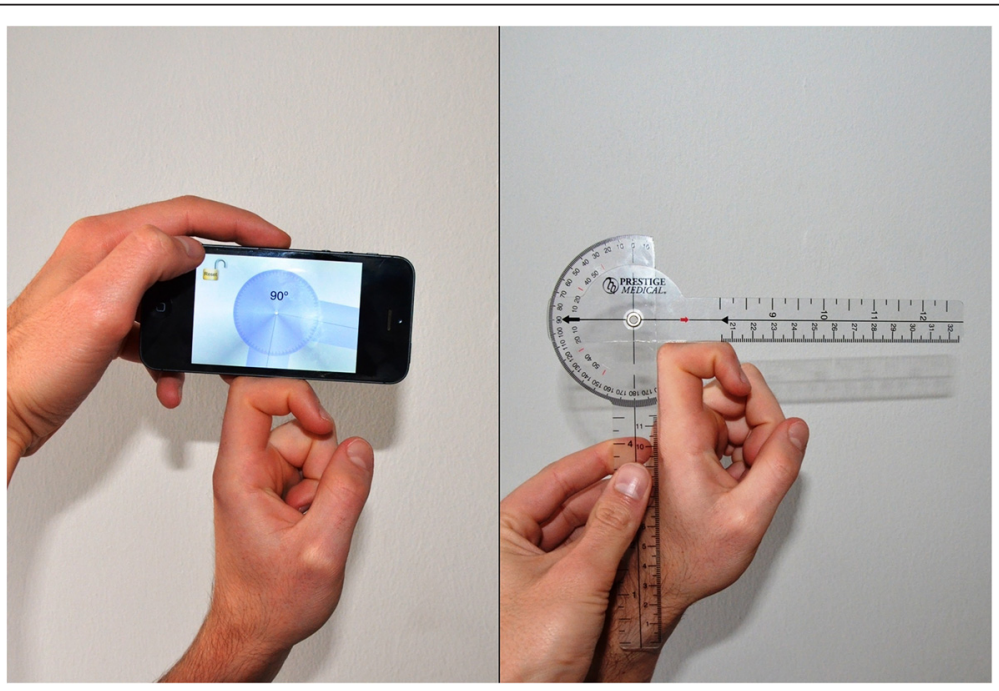

Fig. 3 Left: goniometer-app. Right: mechanical goniometer 
However, we did not intend to evaluate inter-rater variability, and aimed at assessing the apps under optimal standardized conditions. Another limitation was the lack of defined standard angles and distances when taking a picture. Additionally, a weakness of goniometer-apps working with internal sensors in a clinical setting consists in the lack of flat surfaces on a human body: when measuring angles of the pyramid it is easy to align the smartphone along edges and faces, but it becomes more difficult when trying to do so on real joints. Furthermore, we only assessed one type of hardware per operating system, and we cannot exclude that other smartphones might have generated different results based on different equilibration and configuration of the hardware.

\section{Conclusions}

Providing a correct handling of the goniometer-apps, notably correct alignment using either photogoniometers or accelerometer-apps, fourteen out of sixteen apps proved to be reliable and valid in our setting. Each user must be aware that these apps were not approved as Medical Devices, and are therefore subject to each user's own personal responsibility. Apart from being mentioned in the medical literature, none of the tested or cited apps has been readily applied in clinical practice in hand surgery.

Competing interests

The authors declare that they have no competing interests.

\section{Author's contributions}

PK built the pyramid, carried out all measurements, analyzed and interpreted the data, performed the statistical analysis and drafted the manuscript. PW and DBL helped in drafting the manuscript and revised it critically. AT, GS and DBL have been involved in proof-reading and revising the final version of the manuscript. MG drafted the section about legal aspects of goniometerapps regarding the Medical Devices Act. LK designed the study, helped in drafting and revising the manuscript and coordinated the study. All authors read and approved the final manuscript.

\section{Acknowledgements}

There were no others than the authors involved in this study. There was no funding.

\section{Author details}

${ }^{1}$ Division of Plastic, Aesthetic and Reconstructive Surgery, Department of Surgery, Medical University of Graz, Graz, Austria. ${ }^{2}$ Department of Quality and Risk Management, University Hospital Graz, Graz, Austria. ${ }^{3}$ Research Department Medical Informatics, Johannes Kepler University Linz, Linz, Austria.

Received: 29 December 2014 Accepted: 30 April 2015

Published online: 15 July 2015

\section{References}

1. Gartner Says Annual Smartphone Sales Surpassed Sales of Feature Phones for the First Time in 2013 [http://www.gartner.com/newsroom/id/2665715]

2. App Store (iOS)[http://en.wikipedia.org/wiki/App_Store_(iOS)]

3. Google Play [http://en.wikipedia.org/wiki/Google_play]

4. $500 \mathrm{~m}$ people will be using healthcare mobile applications in 2015 [https:// research2guidance.com/500m-people-will-be-using-healthcare-mobileapplications-in-2015/]

5. Mosa AS, Yoo I, Sheets L. A systematic review of healthcare applications for smartphones. BMC Med Inform Decis Mak. 2012;12:67.
6. Payne KB, Wharrad H, Watts K. Smartphone and medical related App use among medical students and junior doctors in the United Kingdom (UK): a regional survey. BMC Med Inform Decis Mak. 2012;12:121.

7. Boruff JT, Storie D. Mobile devices in medicine: a survey of how medical students, residents, and faculty use smartphones and other mobile devices to find information. J Med Libr Assoc. 2014;102(1):22-30.

8. Kulendran M, Lim M, Laws G, Chow A, Nehme J, Darzi A, Purkayastha S. Surgical smartphone applications across different platforms: their evolution, uses, and users. Surg Innov. 2014 Aug;21(4):427-40.

9. Chao EYS. Justification of triaxial goniometer for the measurement of joint rotation. J Biomech. 1980;13(12):989-1006.

10. Tognetti A, Lorussi F, Mura GD, Carbonaro N, Pacelli M, Paradiso R, et al. New generation of wearable goniometers for motion capture systems. J Neuroeng Rehabil. 2014;11(1):56.

11. Ferriero G, Sartorio F, Foti C, Primavera D, Brigatti E, Vercelli S. Reliability of a new application for smartphones (DrGoniometer) for elbow angle measurement. PM R. 2011:3(12):1153-4.

12. Ferriero G, Vercelli S, Sartorio F, Munoz Lasa S, llieva E, Brigatti E, et al. Reliability of a smartphone-based goniometer for knee joint goniometry. Int J Rehabil Res. 2013;36(2):146-51.

13. Mitchell K, Gutierrez SB, Sutton S, Morton S, Morgenthaler A. Reliability and validity of goniometric iPhone applications for the assessment of active shoulder external rotation. Physiother Theory Pract. 2014 Oct;30(7):521-5.

14. Jones A, Sealey R, Crowe M, Gordon S. Concurrent validity and reliability of the Simple Goniometer iPhone app compared with the Universal Goniometer. Physiother Theory Pract. 2014 Oct;30(7):512-6.

15. Ockendon M, Gilbert RE. Validation of a novel smartphone accelerometer-based knee goniometer. J Knee Surg. 2012;25(4):341-5.

16. Jenny JY. Measurement of the knee flexion angle with a Smartphone-application is precise and accurate. J Arthroplasty. 2013;28(5):784-7.

17. Ferriero G, Vercelli S, Sartorio F, Foti C. Accelerometer- and photographicbased smartphone applications for measuring joint angle: are they reliable? J Arthroplasty. 2014;29(2):448-9.

18. Milani P, Coccetta CA, Rabini A, Sciarra T, Massazza G, Ferriero G. Mobile smartphone applications for body position measurement in rehabilitation: a review of goniometric tools. PM R. 2014 Nov;6(11):1038-43.

\section{Submit your next manuscript to BioMed Central and take full advantage of:}

- Convenient online submission

- Thorough peer review

- No space constraints or color figure charges

- Immediate publication on acceptance

- Inclusion in PubMed, CAS, Scopus and Google Scholar

- Research which is freely available for redistribution 